\title{
The Toll-like receptor 4 antagonist TAK-242 protects against chronic pancreatitis in rats
}

\author{
LONG-FEI PAN ${ }^{1}$, LEI YU ${ }^{2}$, LI-MING WANG ${ }^{1}$, JUN-TAO HE ${ }^{3}$, JIANG-LI SUN ${ }^{1}$, XIAO-BO WANG ${ }^{1}$, \\ ZHENG-HAI BAI ${ }^{1}$, HAI WANG ${ }^{1}$, TING-LIN YAN ${ }^{4}$ and HONG-HONG PEI ${ }^{1}$
}

\begin{abstract}
${ }^{1}$ Department of Emergency Medicine, The Second Affiliated Hospital of Xi'an Jiaotong University, Xi'an, Shaanxi 710004; ${ }^{2}$ Department of Basic Medicine, Xi'an Medical College, Xi'an, Shaanxi 710021; ${ }^{3}$ Department of Clinical Laboratory, The Second Affiliated Hospital of Xi'an Jiaotong University, Xi'an, Shaanxi 710004; ${ }^{4}$ Department of Forensic Medicine, Health Science Center of Xi'an Jiaotong University, Xi'an, Shaanxi 710061, P.R. China
\end{abstract}

Received January 26, 2017; Accepted July 4, 2017

DOI: $10.3892 / \mathrm{mmr} .2017 .7105$

\begin{abstract}
Chronic pancreatitis is a progressive disease characterized by irreversible morphological changes to the pancreas, typically causing pain and permanent loss of function. It is a poorly understood disease with the pathogenesis remaining unclear. The authors' previous data demonstrated that the inhibition of Toll-like receptor 4 (TLR4) using TLR4 antagonist kinase (TAK)-242 attenuates taurocholate-induced oxidative stress via the regulation of mitochondrial function in the pancreatic acinar cells of mice. In the present study, the effect of TAK-242 on trinitrobenzene sulfonic acid (TNBS)-induced chronic pancreatitis was investigated in rats. The results revealed that TAK-242 attenuated the severity of chronic pancreatic injury, and regulated extracellular matrix secretion and cellular immunity. In addition, TAK-242 treatment significantly decreased cell apoptosis, as evidenced by the reduction in Terminal deoxynucleotidyl transferase dUTP nick end labeling-positive cells in pancreas tissue sections, and also promoted cell proliferation in TNBS-treated animals. Furthermore, the results of the calibrated von Frey filament assay demonstrated that TAK-242 could prevent the pancreatitis-induced referred abdominal hypersensitivity. In summary, TAK-242 exhibits protective effects against TNBS-induced chronic pancreatitis and may be a potential therapeutic strategy for the treatment of patients with chronic pancreatitis.
\end{abstract}

\section{Introduction}

Chronic pancreatitis is defined as a progressive fibro-inflammatory disease of the pancreas which can be classified into three phenotypes: Chronic calcifying pancreatitis, chronic

Correspondence to: Professor Hong-Hong Pei, Department of Emergency Medicine, The Second Affiliated Hospital of Xi'an Jiaotong University, 157 Xiwu Road, Xi'an, Shaanxi 710004, P.R. China

E-mail: peihonghong_xjtu@126.com

Key words: chronic pancreatitis, TLR4, apoptosis, pain obstructive pancreatitis and chronic auto-immune pancreatitis. The overall incidence of chronic pancreatitis worldwide is approximately 2 to 200 per 100,000, and recent data indicate an increasing trend year by year (1). Current treatments, including internal medicine, endoscopy and surgical intervention, can only relieve symptoms and improve quality of life, but are not effective in halting the advance of the disease (2).

Toll-like receptors (TLRs) are a large family of type I transmembrane proteins that play key roles in innate immunity and inflammatory responses. Thirteen mammalian TLRs have been identified, among which TLR1-TLR10 are conserved between humans and mice (3). Currently known, they are located not only in the immune cells, including B cells, T cells and macrophages, but also in other cells, such as fibroblasts, epithelial cells and pancreatic acinar cells (4). TLR4 is expressed in the internal and external secretory portions of pancreas and has been identified as the signaling receptor for both lipopolysaccharide and endotoxin, the outer membrane components of Gram negative bacteria (5). Previous studies have pointed out the potential role of TLRs in pancreatitis and associated organ injury. For example, TLR4 mRNA was shown to be upregulated in the pancreas of rats in the early stage of pancreatitis (6). The increase in plasma TNF- $\alpha$ levels was found to be positively correlated with the expression of TLR4 in pancreatitis patients (7). Furthermore, our previous data suggested that inhibition of TLR4 attenuates taurocholate-induced oxidative stress via regulating mitochondrial function in mice pancreatic acinar cells (8). In the present study, we investigated the potential protective role of TLR4 antagonist kinase (TAK)-242, a novel TLR4 antagonist, in a chronic pancreatitis model induced by trinitrobenzene sulfonic acid (TNBS) in rats.

\section{Materials and methods}

Subjects. Male Sprague-Dawley rats (250 to $300 \mathrm{~g}$ ) were obtained from the Laboratory Animal Center of the Xi'an Jiaotong University (Xi'an, China). The animals had continuous access to food and water and were housed in cages in a room maintained at 20 to $22^{\circ} \mathrm{C}$ with a $12 \mathrm{~h} \mathrm{light} / 12 \mathrm{~h}$ dark cycle. All experimental protocols and animal handling procedures were 
performed in accordance with the National Institutes of Health (NIH) guidelines for the use of experimental animals and approved by the Institutional Animal Care and Use Committee of the Xi'an Jiaotong University. TAK-242 was dissolved in $1 \%$ DMSO and then diluted in sterile endotoxin-free water.

Chronic pancreatitis model. Induction of chronic pancreatitis was performed according to a previously described method (9). TNBS was dissolved in $10 \%$ ethanol, and animals were intraductally infused with $2 \%$ TNBS in $400 \mu$ l. A same volume of $10 \%$ ethanol in phosphate-buffered saline (PBS) was used to the Vehicle rats.

Measurement of serum amylase, lipase and lactate dehydrogenase $(L D H)$. Blood samples were coagulated after being stored in the refrigerator at $4^{\circ} \mathrm{C}$ overnight. The serum was then collected after being centrifuged at $3,000 \mathrm{~g}$ for $15 \mathrm{~min}$ at $4^{\circ} \mathrm{C}$ and stored at $-80^{\circ} \mathrm{C}$ until use. A medical automatic chemical analyzer was used to determine the serum amylase, lipase and LDH levels.

TUNEL staining. Apoptotic cells in sections of pancreatic tissues were determined using TUNEL staining, a method to observe DNA strand breaks in nuclei. Briefly, $4-\mu \mathrm{m}$ sections were prepared and incubated with TUNEL reaction mixture at $37^{\circ} \mathrm{C}$ for $1 \mathrm{~h}$. The sections were analyzed under a fluorescence microscope at 450-550 $\mathrm{nm}$.

Measurement of pancreatitis-induced pain. Pancreatitis pain was assessed by referred abdominal hypersensitivity to probing the upper left abdominal quadrant of rats with calibrated von Frey filaments (10). Before testing, rats were allowed to acclimate in a suspended wire-mesh cage for $30 \mathrm{~min}$ and the belly skin of the rat was shaved and area designated for stimulation was marked. The von Frey filaments were applied from underneath through the mesh floor, in ascending order to the abdominal area at different points on the surface. A single trial consisted of 5 applications of a von Frey filament applied once every $10 \mathrm{sec}$. A positive response consisted of sharp withdrawal of the abdomen, licking of abdominal area, or whole body withdrawal. The mean occurrence of withdrawal events in each trial is expressed as the number of responses to 5 applications.

Western blot analysis. Equivalent amounts of protein $(40 \mu \mathrm{g}$ per lane) were loaded and separated by $10 \%$ SDS-PAGE gels, and transferred to polyvinylidene difluoride membranes. Membranes were blocked with 5\% non-fat milk solution in Tris-buffered saline with $0.1 \%$ Triton X-100 (TBST) for $1 \mathrm{~h}$, and then incubated overnight at $4^{\circ} \mathrm{C}$ with the primary collagen $1 \alpha 1, \alpha$-smooth muscle actin ( $\alpha$-SMA), TGF- $\beta$, matrix metalloproteinase (MMP)-2, MMP-9, CD68, CD3, CD4, CD8, CD19, amylase, PCNA, caspase-3, Bcl-2, Bax, and $\beta$-actin antibody dilutions in TBST. After that the membranes were washed and incubated with secondary antibody for $1 \mathrm{~h}$ at room temperature. Immunoreactivity was detected with Super Signal West Pico Chemiluminescent Substrate (Thermo Fisher Scientific Inc., Rockford, IL, USA). An analysis software named Image J (Scion Corporation, Bethesda, MA, USA) was used to quantify the optical density of each band.
Statistical analysis. Statistical analysis was performed using SPSS 16.0 (SPSS, Inc., Chicago, IL, USA), a statistical software package. Statistical evaluation of the data was performed by one-way analysis of variance (ANOVA) followed by Bonferroni's multiple comparisons. A value of $\mathrm{P}<0.05$ was considered statistically significant.

\section{Results}

TAK-242 attenuates the severity of chronic pancreatitis. To mimic chronic pancreatitis in vivo, rats were treated with $400 \mu 1$ TNBS by intraductal infusion, and significant decreases in body weight and pancreas weight were detected in Vehicle group. Treatment with TAK-242 markedly increased the body weight compared with that in Vehicle-treated animals in week 5 and 6, but not in week 4 (Fig. 1A). As shown in Fig. 1B, TNBS-induced pancreas weight loss was ameliorated by TAK-242. Blood samples were collected at week 5 and analyzed by the automatic biochemical analyzer. Neither TNBS nor TAK-242 has effect on the serum levels of amylase and lipase (Fig. 1C and D), whereas TAK-242 partially prevented the TNBS-induced elevation of serum LDH level (Fig. 1E).

TAK-242 modulates extracellular matrix (ECM) secretion and cellular immunity. Western blot analysis was performed to quantify the expression of ECM associated proteins (Fig. 2). The expression of collagen $1 \alpha 1, \alpha$-SMA and TGF- $\beta$ increased obviously after TNBS administration compared with control group but decreased after TAK-242 treatment. TAK-242 also partially prevented the TNBS-induced upregulation of MMP-9, whereas the increased expression of MMP-2 was not significantly affected by TAK-242. In addition, we detected the expression of CD68, CD3, CD4, CD8 and CD19 to investigate the effect of TAK-242 on the cellular immunity in chronic pancreatitis (Fig. 3A). As shown in Fig. 3B, all these proteins were obviously upregulated after TNBS administration, and the application of TAK-242 markedly reduced the expression of these proteins.

TAK-242 modulates the proliferation and apoptosis in chronic pancreatitis. It is well known that both cellular apoptosis and proliferation are involved in the chronic pancreatic injury induced by TNBS. Thus, we detected the expression of proliferation and apoptosis related proteins by western blot analysis (Fig. 4A). As shown in Fig. 4B, TNBS decreased the expression of amylase, and TAK-242 significantly preserved the expression of amylase in TNBS-treated animals. In contrast, the expression of PCNA was upregulated after TNBS administration, which was further promoted by TAK-242. In addition, TAK-242 treatment significantly decreased the expression of Bax and caspase-3, but increased the expression of Bcl-2 after TNBS administration. We also performed the TUNEL staining to detect apoptosis in vivo, and the results indicated increased acinar cell apoptosis in mice following the induction of chronic pancreatitis (Fig. 4C). The number of TUNEL positive cells was lower after TAK-242 treatment than that in TNBS group.

TAK-242 prevents pancreatitis-induced referred abdominal hypersensitivity. Pancreatitis-induced pain was evaluated by probing the abdomen with von Frey filaments, and obvious abdominal hypersensitivity in TNBS-treated animals was 
A
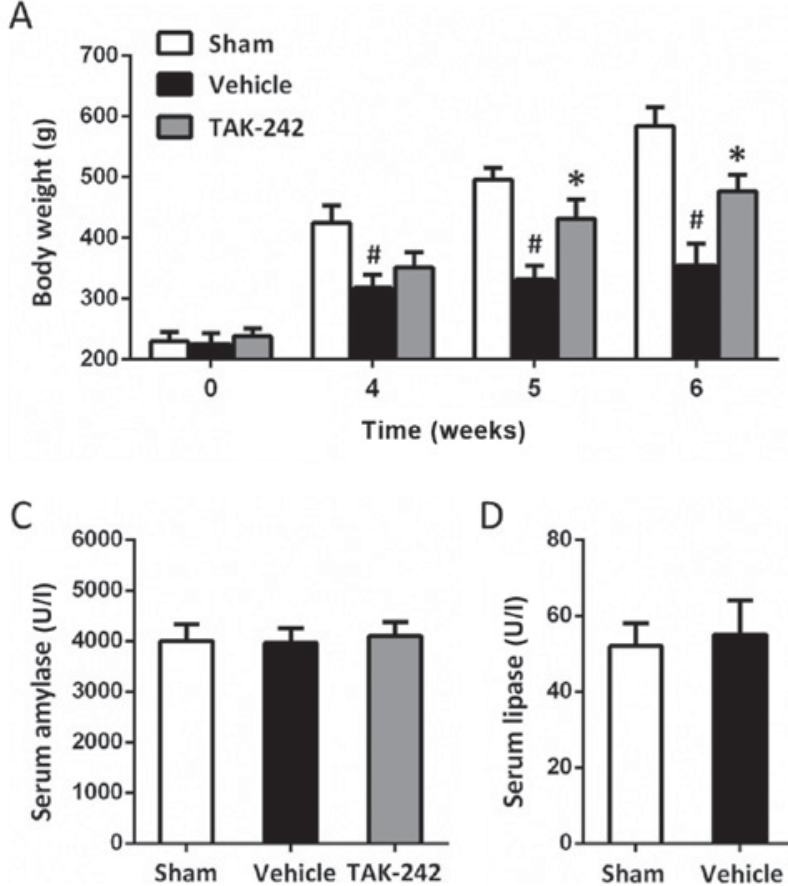

B

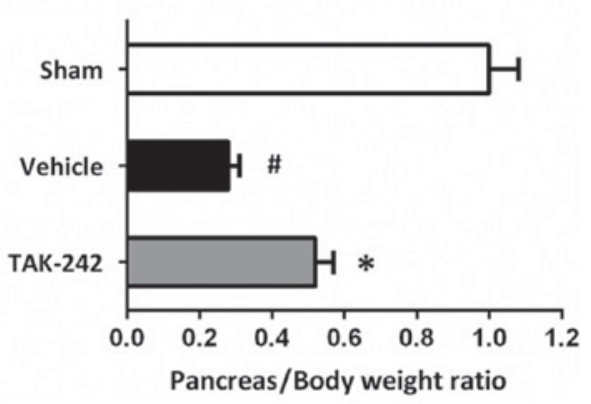

$\mathrm{E}$

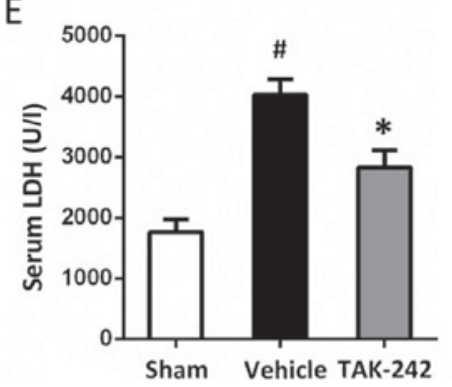

Figure 1. TAK-242 attenuates the severity of chronic pancreatitis. The animals were intraperitoneally treated with $3 \mathrm{mg} / \mathrm{kg}$ TAK-242 or $1 \%$ DMSO (Vehicle) and treated with $400 \mu 1$ TNBS by intraductal infusion. (A) The body weight was measured at 0, 4, 5 and 6 weeks later, and (B) the pancreas weight was measured at 6 weeks later. The serum (C) amylase, (D) lipase and (E) LDH were measured, respectively. Data are shown as mean \pm SEM $(\mathrm{n}=8)$. ${ }^{~} \mathrm{P}<0.05$ vs. Sham group. " $\mathrm{P}<0.05$ vs. Vehicle group. TAK, Toll-like receptor 4 antagonist kinase; TNBS, trinitrobenzene sulfonic acid; LDH, lactate dehydrogenase.
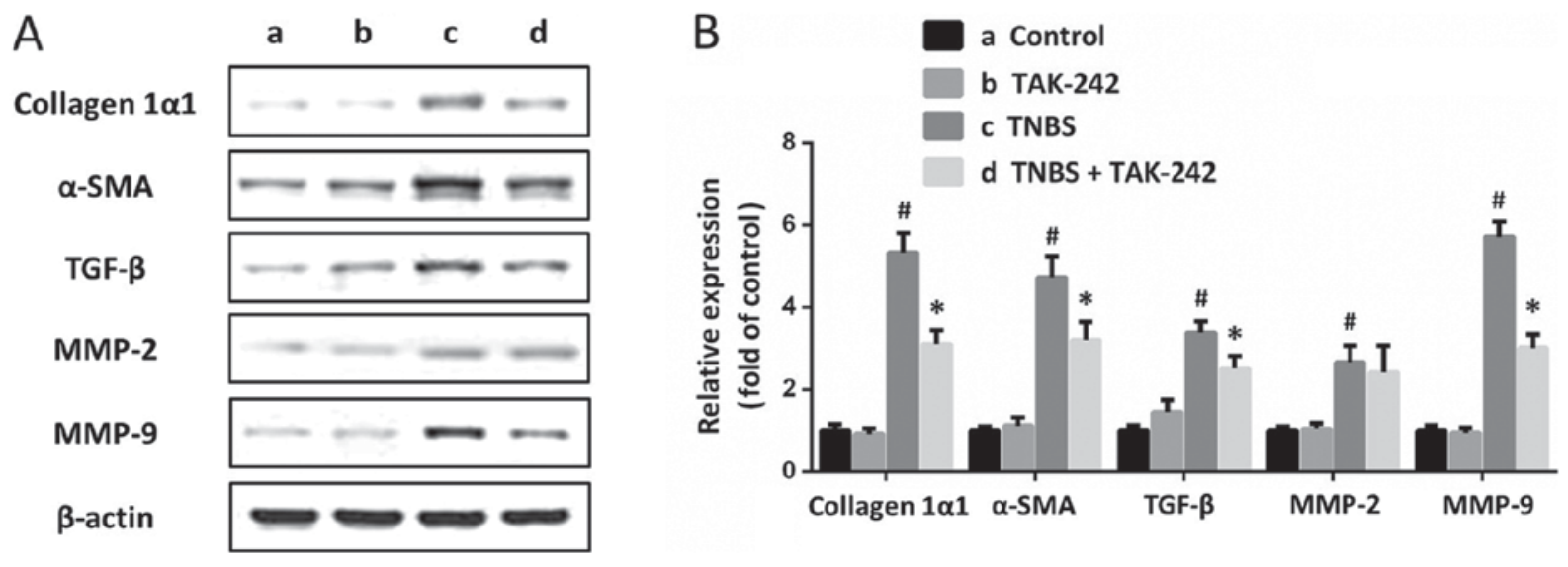

Figure 2. TAK-242 modulates the extracellular matrix secretion in chronic pancreatitis. The animals were treated with $400 \mu 1$ TNBS by intraductal infusion with or without intraperitoneally administration of $3 \mathrm{mg} / \mathrm{kg}$ TAK-242. The expression of extracellular matrix associated proteins was detected (A) by western blot analysis and (B) calculated on week 6 . Data are shown as mean \pm SEM $(n=8) .{ }^{~} \mathrm{P}<0.05$ vs. control group. ${ }^{*} \mathrm{P}<0.05$ vs. TNBS group. TNBS, trinitrobenzene sulfonic acid; TAK, Toll-like receptor 4 antagonist kinase; $\alpha$-SMA, $\alpha$-smooth muscle actin; MMP, matrix metalloproteinase.

proved by the increased number of responses to 5 applications of von Frey filaments. The concentration of TAK- 242 as $3 \mathrm{mg} / \mathrm{kg}$ was chosen according to a previous paper (11). As shown in Fig. 5A, a dose-dependent reversal of response number was observed after TAK-242 treatment. In addition, the responses in TAK-242 treated chronic pancreatitis rats were significantly reduced and were markedly different from rats treated with TNBS alone in week 5 and 6, but not in week 4 (Fig. 5B).

\section{Discussion}

Chronic pancreatitis is characterized by inflammation and fibrosis, and one of the most important mechanisms of this process is the activation of innate immune system. In our study of chronic pancreatitis induced by TNBS, we demonstrated the protective effect of blocking TLR4, an important regulator of immune/inflammatory responses. The pathophysiologic changes present in the pancreas during chronic pancreatitis are complicated. Our results showed that TAK-242 attenuates pancreas weight loss, modulates ECM secretion, regulates cellular immunity and reduces acinar cell apoptosis. In addition, TAK-242 administration also prevents pancreatitis-induced referred abdominal hypersensitivity, the most common and typical clinical symptom of patients with chronic pancreatitis.

TLR4 was originally identified to generate innate immune responses to pathogens by activating pro-inflammatory 

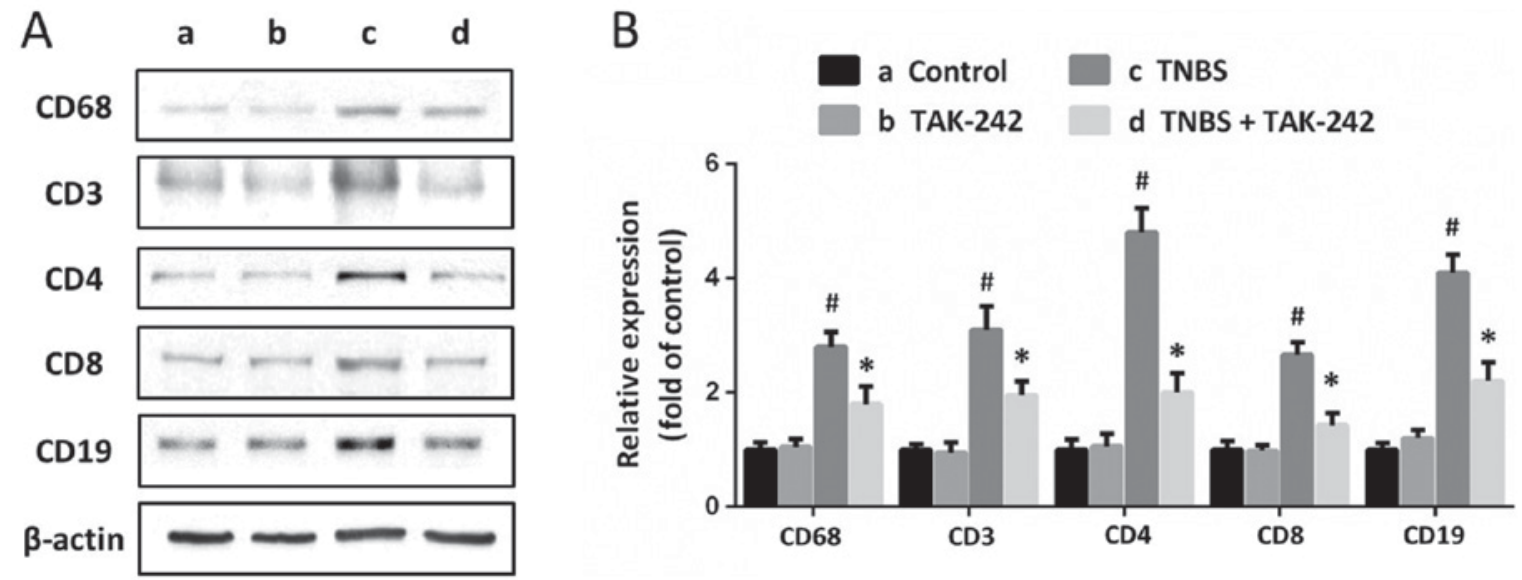

Figure 3. TAK-242 regulates the cellular immunity in chronic pancreatitis. The animals were treated with $400 \mu 1$ TNBS by intraductal infusion with or without intraperitoneally administration of $3 \mathrm{mg} / \mathrm{kg}$ TAK-242. The expression of CD68, CD3, CD4, CD8 and CD19 proteins were detected (A) by western blot and (B) calculated on week 6 . Data are shown as mean \pm SEM ( $n=8)$. ${ }^{~} \mathrm{P}<0.05$ vs. control group. ${ }^{*} \mathrm{P}<0.05$ vs. TNBS group. TAK, Toll-like receptor 4 antagonist kinase; TNBS, trinitrobenzene sulfonic acid.
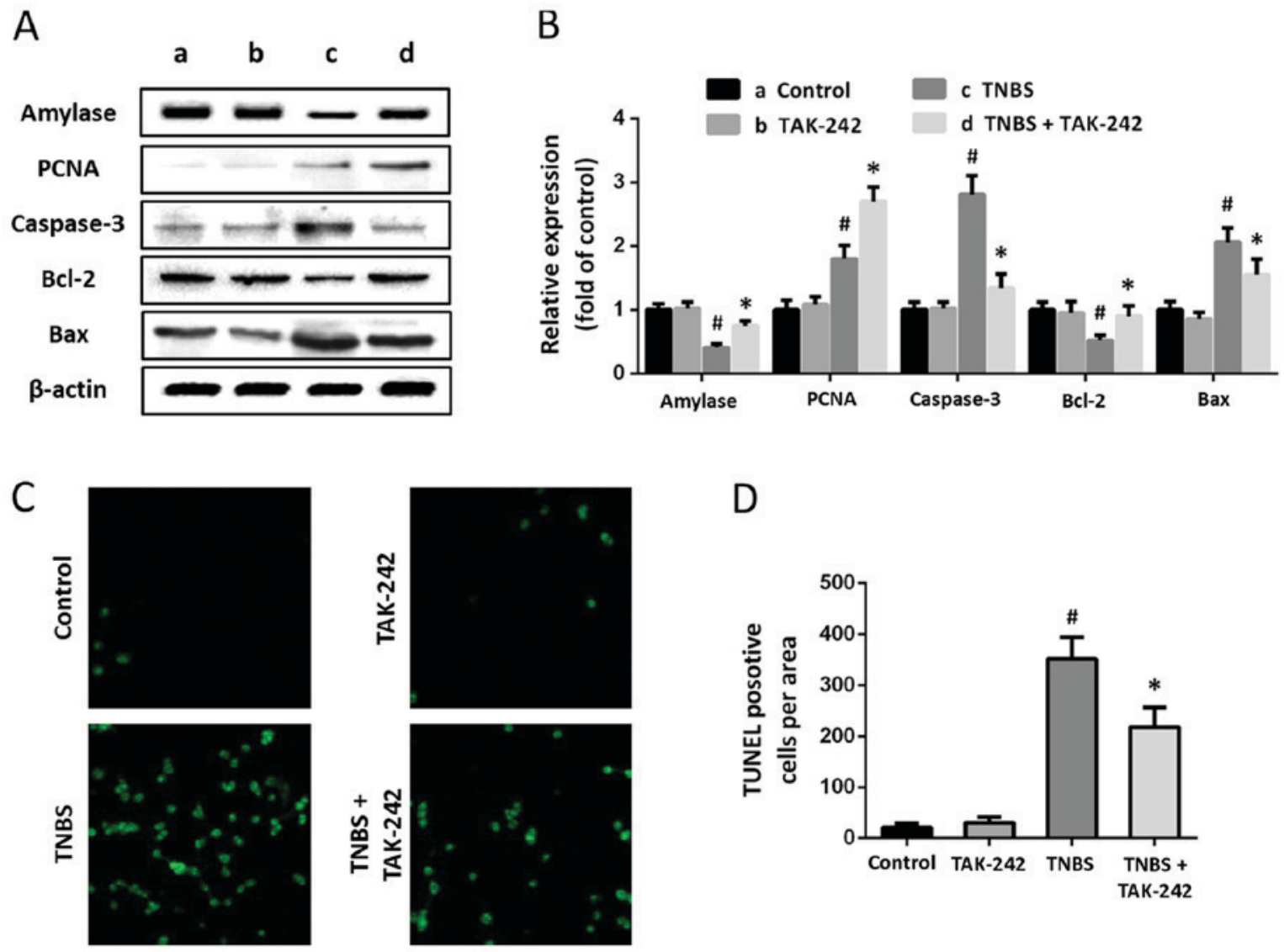

Figure 4. TAK-242 modulates the proliferation and apoptosis in chronic pancreatitis. The animals were treated with $400 \mu 1$ TNBS by intraductal infusion with or without intraperitoneally administration of $3 \mathrm{mg} / \mathrm{kg}$ TAK-242. The expression of amylase, PCNA, caspase-3, Bcl-2 and Bax proteins were detected (A by western blot and (B) calculated on week 6. (C) The apoptotic cell death in pancreas sections was detected by TUNEL staining, and (D) the number of TUNEL-positive cells was counted. Data are shown as mean \pm SEM $(n=8) .{ }^{~} \mathrm{P}<0.05$ vs. control group. ${ }^{*} \mathrm{P}<0.05$ vs. TNBS group. TAK, Toll-like receptor 4 antagonist kinase; TNBS, trinitrobenzene sulfonic acid.

events (12). Significant findings on TLR4-mediated signaling cascades in inflammatory diseases have been reported, such as upregulation of plasma TNF- $\alpha$ and nuclear factor- $\kappa \mathrm{B}(\mathrm{NF}-\kappa \mathrm{B})$ levels (7). Previous studies showed that TLR4 could recognize its endogenous ligands and activate macrophages to release multiple inflammatory mediators during acute pancreatitis (13).
TAK-242 is an exogenous synthetic antagonist for TLR4, which can inhibit TLR4 signaling and attenuate the inflammatory responses under pathological conditions (14). Our previous study found that TAK-242 was effective in ameliorating cytotoxicity and apoptosis induced by taurocholate exposure in a cellular model of acute pancreatitis (8). The protective 
A

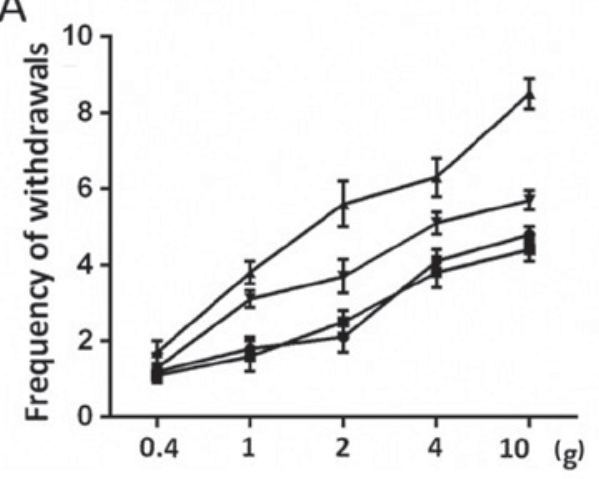

B

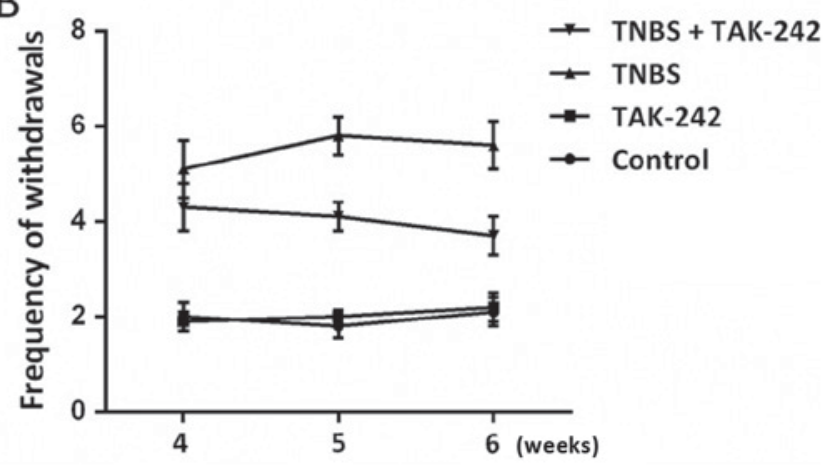

Figure 5. TAK-242 prevents pancreatitis-induced referred abdominal hypersensitivity. The animals were treated with $400 \mu 1 \mathrm{TNBS}$ by intraductal infusion with or without intraperitoneally administration of $3 \mathrm{mg} / \mathrm{kg}$ TAK-242. Pancreatitis-induced pain was evaluated by probing the abdomen with von Frey filaments at 4, 5 and 6 weeks after the induction of chronic pancreatitis by TNBS. (A) A dose-dependent reversal of referred abdominal hypersensitivity was produced by TAK-242 in TNBS-induced chronic pancreatitis on week 6. (B) A time-dependent reversal of referred abdominal hypersensitivity was produced by TAK-242 in TNBS-induced chronic pancreatitis. Data are shown as mean \pm SEM $(n=8)$. TAK, Toll-like receptor 4 antagonist kinase; TNBS, trinitrobenzene sulfonic acid.

effects were associated with inhibited oxidative stress and preserved mitochondrial function. Here, we extended the protective effect of TAK-242 into an animal model of chronic pancreatitis. These results are helpful for understanding the possible roles of TLR4 in pancreatitis and will help to better understand the pathogenesis in chronic pancreatitis.

Fibrogenesis is a characteristic pathological alteration of chronic pancreatitis, and the prolonged activation of fibrotic cascades will eventually lead to the loss of pancreatic endocrine and exocrine functions (15). The most crucial step of pancreatic fibrosis is the activation of pancreatic stellate cells (PSCs), which transform into an activated phenotype and secrete excessive amounts of ECM proteins, such as $\alpha$-SMA, collagens and MMPs $(16,17)$. In TNBS-induced pancreatitis, activated PSCs were shown to be associated with the main cellular of collagen and the positive areas of $\alpha$-SMA-stained sections (9). As shown in Fig. 2, activated PSCs and ECM deposition were identified by the increased expression of collagen-1 $\alpha 1$ and $\alpha$-SMA, indicating the pancreatic fibrosis after TNBS treatment in our model. Another key factor that influences pancreatic fibrosis in chronic pancreatitis is the balance of ECM synthesis and degradation. Among multiple inflammatory cytokines, TGF- $\beta$ acts at different levels to increase pancreatic collagen deposition $(18,19)$. In addition, the serum levels of MMP-9 were increased in patients with recurrent chronic pancreatitis (20), and the active form of MMP-9 was associated with the development of diabetes mellitus in chronic pancreatitis (21). In the present study, we found that TAK-242 partially prevented the TNBS-induced increase in collagen- $1 \alpha 1, \alpha$-SMA, TGF- $\beta$, MMP-2 and MMP-9 expression, indicating that TLR4 blockage might inhibit pancreatic fibrosis via modulating ECM deposition.

The mechanism of pancreatic acinar cell death and survival in chronic pancreatitis has not been extensively understood. It was well accepted that the process of cellular apoptosis and proliferation were both participated, but their relationship with the progression of chronic pancreatitis remains unknown. Previous studies have shown that the acinar cell apoptosis index and proliferation index were higher in chronic pancreatitis than controls (22). In consistent, our results of western blot analysis showed increased expression of PCNA, caspase-3 and bax, as well as decreased expression of amylase, and $\mathrm{Bcl}-2$ after TNBS treatment. A recent study found that recombinant interleukin-1 receptor antagonist attenuates the severity of chronic pancreatitis induced by TNBS through promoting cellular proliferation and inhibiting apoptosis (9). As an antagonist for TLR4, TAK-242 has been shown to regulate cellular apoptosis in multiple human diseases $(11,23)$. Our previous experiments showed that TAK-242 attenuated taurocholate-induced oxidative stress through regulating mitochondrial associated apoptosis in mice pancreatic acinar cells (8). Here, we found that TNBS-induced alteration in the expression of proliferation and apoptosis related proteins and increased TUNEL positive cells were both partially prevented by TAK-242. Therefore, we speculated that TAK-242-induced protection might be associated with reduction of acinar cell loss mediated by apoptosis inhibition, as well as enhanced pancreas regeneration by improvement of cellular proliferation in the pancreas.

Although typical clinical symptom of patients with chronic pancreatitis, such as weight loss, steatorrhea, diabetes and malnutrition, are vague and not specific, approximately $90 \%$ of patients suffer from abdominal pain (24). The pancreatic pain is usually epigastric, dull and constant in nature, and always radiate to the back or laterally to the left and right flank (25). It was originally considered to be caused by anatomical changes in pancreatic structure, but strategies based on this theory are shown unsatisfactory. Increasing evidence indicate neurobiological theories for the explanation of pain in chronic pancreatitis, and multiple inflammatory mediators, such as prostanoid, tachykinin, and growth factors, as well as neurotransmitters, including glutamate and substance $\mathrm{P}$, are thought to be in involved in these mechanisms $(26,27)$. As the 'gate' of inflammatory response, TLR4 has been shown to play a key role in chronic pain states of somatic origin in preclinical models. Intrathecal injection of short interfering RNA (siRNA) against TLR4 reduced nociception in a rat model of neuropathic pain, and downregulation of TLR4 gene expression by siRNA attenuated bone cancer pain in a rat model $(28,29)$. Using pharmacological and genetic approaches, Tramullas and et al demonstrated that TLR4 was required to modulate visceral pain under physiological conditions, and pharmacological blockade of TLR4 was able to 
counteract the hyper-responsive phenotype in an animal model of stress-induced visceral hypersensitivity (30). In the present study, we found that the TLR4 antagonist TAK-242 produced a dose- and time-dependent reversal of referred abdominal hypersensitivity. We speculated that peripheral administration of TAK-242 might blunt abdominal hypersensitivity through reducing release of inflammatory cytokines, or it could impede the sensory information transmission from the gastrointestinal tract to the central nervous system, which needs to be further determined.

In summary, our present data can be interpreted to show that the TLR4 antagonist TAK-242 is effective in attenuating the severity of TNBS-induced chronic pancreatitis. This effect is associated with modulation of ECM secretion, regulation of cellular immunity and inhibition of cellular apoptosis.

\section{Acknowledgements}

The present study was financially supported by the Project Fund from Science and Technology Project of Shaanxi Province (no. 2010K15-03-02), Shaanxi Province Key Scientific and Technological Project (no. 2016SF-232) and Scientific Research Fund Project of Shaanxi Provincial Health and Family Planning Commission (no. 2016D015). The authors would like to thank Dr Terry Chen for his technical support for the experiments and the preparation of the manuscript.

\section{References}

1. Ni Q, Yun L, Roy M and Shang D: Advances in surgical treatment of chronic pancreatitis. World J Surg Oncol 13: 34, 2015.

2. Conwell DL, Lee LS, Yadav D, Longnecker DS, Miller FH, Mortele KJ, Levy MJ, Kwon R, Lieb JG, Stevens T, et al: American pancreatic association practice guidelines in chronic pancreatitis: Evidence-based report on diagnostic guidelines. Pancreas 43: 1143-1162, 2014.

3. Akira S: Toll-like receptors and innate immunity. Adv Immunol 78: 1-56, 2001.

4. Wang JY, Wen LL, Huang YN, Chen YT and Ku MC: Dual effects of antioxidants in neurodegeneration: Direct neuroprotection against oxidative stress and indirect protection via suppression of glia-mediated inflammation. Curr Pharm Des 12: $3521-3533,2006$

5. Trotta T, Porro C, Calvello R and Panaro MA: Biological role of Toll-like receptor-4 in the brain. J Neuroimmunol 268: 1-12, 2014.

6. Li Y, Zhou ZG, Xia QJ, Zhang J, Li HG, Cao GQ, Wang R, Lu YL and $\mathrm{Hu}$ TZ: Toll-like receptor 4 detected in exocrine pancreas and the change of expression in cerulein-induced pancreatitis. Pancreas 30: 375-381, 2005.

7. Li HG, Zhou ZG, Li Y, Zheng XL, Lei S, Zhu L and Wang Y: Alterations of Toll-like receptor 4 expression on peripheral blood monocytes during the early stage of human acute pancreatitis. Dig Dis Sci 52: 1973-1978, 2007.

8. Pan LF, Yu L, Wang LM, He JT, Sun JL, Wang XB, Bai ZH, Su LJ and Pei HH: The toll-like receptor 4 antagonist transforming growth factor-b-activated kinase(TAK)-242 attenuates taurocholate-induced oxidative stress through regulating mitochondrial function in mice pancreatic acinar cells. J Surg Res 206: 298-306, 2016.

9. Xu C, Shen J, Zhang J, Jia Z, He Z, Zhuang X, Xu T, Shi Y, Zhu S, Wu M and Han W: Recombinant interleukin-1 receptor antagonist attenuates the severity of chronic pancreatitis induced by TNBS in rats. Biochem Pharmacol 93: 449-460, 2015.

10. Vardanyan M, Melemedjian OK, Price TJ, Ossipov MH, Lai J, Roberts E, Boos TL, Deschamps JR, Jacobson AE, Rice KC and Porreca F: Reversal of pancreatitis-induced pain by an orally available, small molecule interleukin-6 receptor antagonist. Pain 151: 257-265, 2010.
11. Hua F, Tang H, Wang J, Prunty MC, Hua X, Sayeed I and Stein DG: TAK-242, an antagonist for Toll-like receptor 4, protects against acute cerebral ischemia/reperfusion injury in mice. J Cereb Blood Flow Metab 35: 536-542, 2015.

12. Medzhitov R: Toll-like receptors and innate immunity. Nat Rev Immunol 1: 135-145, 2001.

13. Zhang X, Zhu C, Wu D and Jiang X: Possible role of toll-like receptor 4 in acute pancreatitis. Pancreas 39: 819-824, 2010.

14. Rice TW, Wheeler AP, Bernard GR, Vincent JL, Angus DC, Aikawa N, Demeyer I, Sainati S, Amlot N, Cao C, et al: A randomized, double-blind, placebo-controlled trial of TAK-242 for the treatment of severe sepsis. Crit Care Med 38: 1685-1694, 2010.

15. Apte M, Pirola R and Wilson J: The fibrosis of chronic pancreatitis: New insights into the role of pancreatic stellate cells. Antioxid Redox Signal 15: 2711-2722, 2011.

16. Moore MJ, Hamm J, Dancey J, Eisenberg PD, Dagenais M, Fields A, Hagan K, Greenberg B, Colwell B, Zee B, et al: Comparison of gemcitabine versus the matrix metalloproteinase inhibitor BAY 12-9566 in patients with advanced or metastatic adenocarcinoma of the pancreas: A phase III trial of the National Cancer Institute of Canada Clinical Trials Group. J Clin Oncol 21: 3296-3302, 2003.

17. Bodey B, Bodey B Jr, Siegel SE and Kaiser HE: Immunocytochemical detection of the expression of members of the matrix metalloproteinase family in adenocarcinomas of the pancreas. In Vivo 15: 71-76, 2001.

18. Bartram U and Speer CP: The role of transforming growth factor beta in lung development and disease. Chest 125: 754-765, 2004.

19. Menke A and Adler G: TGFbeta-induced fibrogenesis of the pancreas. Int J Gastroint Cancer 31: 41-46, 2002.

20. Akhmedov VA, Budylgin AV and Dolgikh VT: The matrix metalloproteinase 9 (MMP-9) and TIMP-1 activities in patients with chronic and recurrent pancreatitis. Eksp Klin Gastroenterol: 11-13, 2010 (In Russian).

21. Descamps FJ, Van den Steen PE, Martens E, Ballaux F, Geboes K and Opdenakker G: Gelatinase B is diabetogenic in acute and chronic pancreatitis by cleaving insulin. FASEB J 17: 887-889, 2003.

22. Bateman AC, Turner SM, Thomas KS, McCrudden PR, Fine DR, Johnson PA, Johnson CD and Iredale JP: Apoptosis and proliferation of acinar and islet cells in chronic pancreatitis: Evidence for differential cell loss mediating preservation of islet function. Gut 50: 542-548, 2002.

23. Zhang Y, Peng W, Ao X, Dai H, Yuan L, Huang X and Zhou Q: TAK-242, a toll-like receptor 4 antagonist, protects against aldosterone-induced cardiac and renal injury. PLoS One 10: e0142456, 2015.

24. Andren-Sandberg A, Hoem D and Gislason H: Pain management in chronic pancreatitis. Eu J Gastroenterol Hepatol 14: 957-970, 2002.

25. Duggan SN, Ni Chonchubhair HM, Lawal O, O'Connor DB and Conlon KC: Chronic pancreatitis: A diagnostic dilemma. World J Gastroenterol 22: 2304-2313, 2016.

26. Atsawarungruangkit A and Pongprasobchai S: Current understanding of the neuropathophysiology of pain in chronic pancreatitis. World J Gastrointest Pathophysiol 6: 193-202, 2015.

27. Vardanyan M and Rilo HL: Pathogenesis of chronic pancreatitis-induced pain. Discov Med 9: 304-310, 2010.

28. Wu FX, Bian JJ, Miao XR, Huang SD, Xu XW, Gong DJ, Sun YM, Lu ZJ and Yu WF: Intrathecal siRNA against Toll-like receptor 4 reduces nociception in a rat model of neuropathic pain. Int J Med Sci 7: 251-259, 2010.

29. Lan LS, Ping YJ, Na WL, Miao J, Cheng QQ, Ni MZ, Lei L, Fang LC, Guang RC, Jin Z and Wei L: Down-regulation of Toll-like receptor 4 gene expression by short interfering RNA attenuates bone cancer pain in a rat model. Mol Pain 6: 2, 2010.

30. Tramullas M, Finger BC, Moloney RD, Golubeva AV, Moloney G, Dinan TG and Cryan JF: Toll-like receptor 4 regulates chronic stress-induced visceral pain in mice. Biol Psychiatry 76: 340-348, 2014. 